\title{
A Laser Radar with High Precision Ranging based on Phase
}

\author{
Lianpeng $\mathrm{Li}^{1, \mathrm{a}, *}$, Zhong $\mathrm{Su}^{2, \mathrm{~b}}$, Lun $\mathrm{Xie}^{1, \mathrm{c}}$ and Xun $\mathrm{Zhao}^{3, \mathrm{~d}}$ \\ ${ }^{1}$ University of Science and Technology Beijing, 100083, Beijing, China \\ ${ }^{2}$ University of Beijing Information Science \& Technology, 100101, Beijing, China \\ ${ }^{3}$ Beijing Institute of Technology, 100081, Beijing, China \\ âB20170311@xs.ustb.edu.cn, bz@bistu.edu.cn, , ${ }^{\text {b } x i e l u n @ u s t b . e d u . c n, ~}{ }^{\mathrm{d}}$ zhaoxu00@126.com \\ * corresponding author
}

Keywords: Laser radar, Phase, Machine learning, Laser ranging.

\begin{abstract}
Laser radar is a radar system that uses laser as light source and works in the optical band. It is the product of the combination of laser technology and radar technology. It has the advantages of all-weather, high precision, anti-interference and miniaturization. Aiming at the great potential of laser radar, a laser radar system based on phase high-precision ranging is proposed. And aiming at the stability of output power caused by temperature variation of semiconductor laser, proposed a laser radar voltage matching algorithm based on machine learning. Through the experiment simulation and functional test, it is proved that the detection distance of the proposed laser beam can reach $300 \mathrm{~m}$ with $0.1 \%$ precision, and it can greatly reduce the influence of temperature change on ranging, and significantly improve the ranging accuracy.
\end{abstract}

\section{Introduction}

Lidar is a radar system that combines traditional radar technology with modern laser technology and works with spectrum. Due to the unique physical properties of laser radar, its all-weather, high precision, anti-interference, miniaturization and other unique advantages, laser ranging has attracted more and more attention, and has become an important technical means in the field of space detection. It has high application value in military and industrial fields ${ }^{[1]}$.

In the second years since the advent of the laser, scientists and engineers abroad have proposed the idea of laser ranging, laser radar and laser guidance. Many research institutes and companies have carried out the development of laser radar ranging system. In recent years, more and more foreign companies are devoted to the development of laser radar series products. Among them, the more famous companies are the German mainland, Germany Sick, Germany IBEO and the American Velodyne company and so on ${ }^{[2]}$. The main units of laser radar research in China are China Electronics Group Corporation 27, Harbin Institute of Technology, Zhejiang University, national defense science and Technology University and Shanghai Institute of technology physics. Overall, the gap between domestic and international is obvious ${ }^{[3]}$. In China, laser radar ranging technology is still in its infancy, only a few laser radar products are available, most of them are in the laboratory stage. At present, the function is basically limited to range ranging. If the range is not accurate, a lot of further signal processing cannot be carried out. Moreover, the semiconductor laser is difficult to maximize its performance due to the influence of temperature changes ${ }^{[4,5]}$. Therefore, In order to improve the detection ability of laser radar and realize the high precision ranging of laser radar, a new phase laser radar is proposed in this paper, also a machine learning laser radar matching algorithm is proposed for the influence of temperature variation on laser radar. 


\section{System Composition and Working Principle}

\subsection{System Composition}

As shown in Figure 1, high precision ranging laser radar based on phase type mainly consists of laser, detector, amplifier (preamplifier and amplifier), processor, power module, temperature module and communication module.

The laser signal is transmitted through the laser modulation module through the optical antenna by the optical tube window. Aiming light path parallel to target scanning. The optical signal received from the target is received by the receiving optical antenna and then amplified by the imaging optical system on the detector and converted into electrical signals. After the signal is converted into the main processor through the analog to digital conversion, the main processor processes the electrical signals and extracts the target distance, velocity information and target image information.

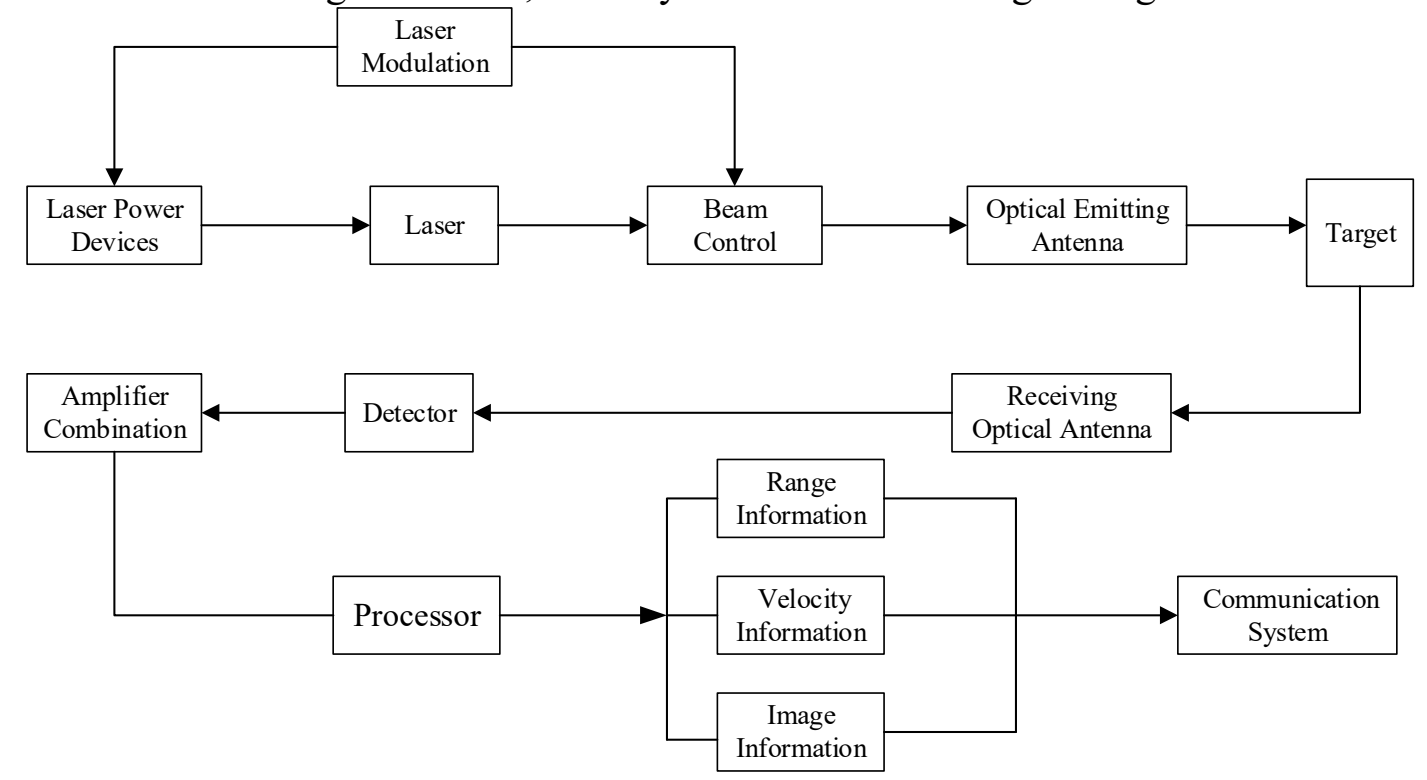

Figure 1 Working principle of laser radar

\subsection{Laser Emission and Detection Module}

The laser emission module mainly includes laser and drive circuit. The driving circuit receives the trigger signal from the signal processor, and drives the laser to emit a peak power of $40 \mathrm{~W}$ and a laser with a wavelength of $905 \mathrm{~nm}$. After the laser encounters the target, diffuse reflection occurs, and the echo signal enters the detection module. The detection module mainly includes detector and drive circuit. The optical signal is focused on the photodiode and converted into electrical signal through the lens, and then transmitted to processor for processing.

The laser transmitting lens is parallel with the optical axis of the detecting lens, and the stability of the optical system is taken into full consideration. The laser emission module and the detection module are fixed and unified on the shell body, as shown in Figure 3.

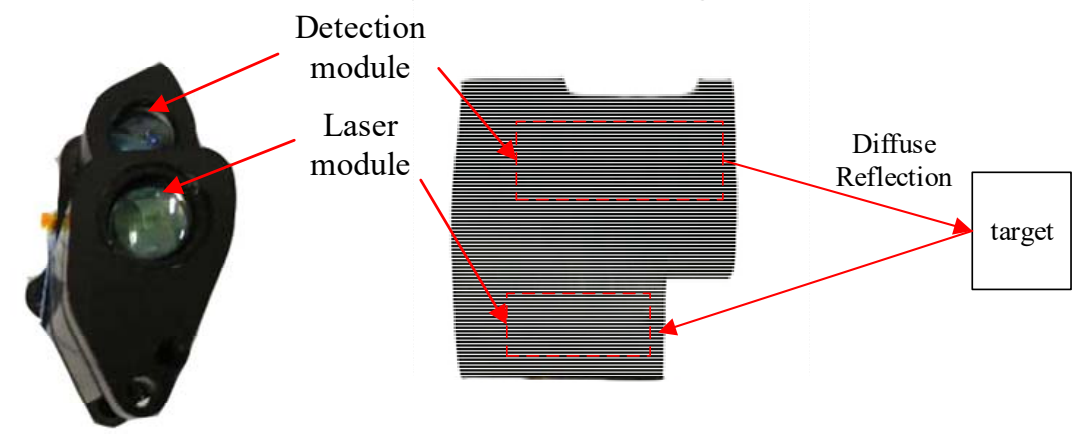

Figure 2 Laser emission and detection module 


\subsection{Signal Processing}

The STM32F4 series of STMicroelectronics is used as the core component of laser radar. The STM32F4 series uses a $90 \mathrm{~nm}$ NVM process and ART, and Microcontrollers incorporate single cycle DSP instructions and FPU. The computational ability is improved, and some complex calculation and control can be carried out. Ultra-fast data transmission, integrated single precision FPU, enhance the execution speed and code efficiency of control algorithm, up to 1M bytes of on-chip flash memory, 192K bytes of SRAM, reset circuit, internal RC, PLL, 1uA RTC ${ }^{[6]}$.

The block diagram of the signal processing system is shown in Figure 3. The detector will detect the modulated optical signal by photoelectric conversion into a current signal, after current to voltage amplification into a voltage signal after amplification, filtering to remove the noise in the signal processor for calculating the phase difference, the distance of output data signal.

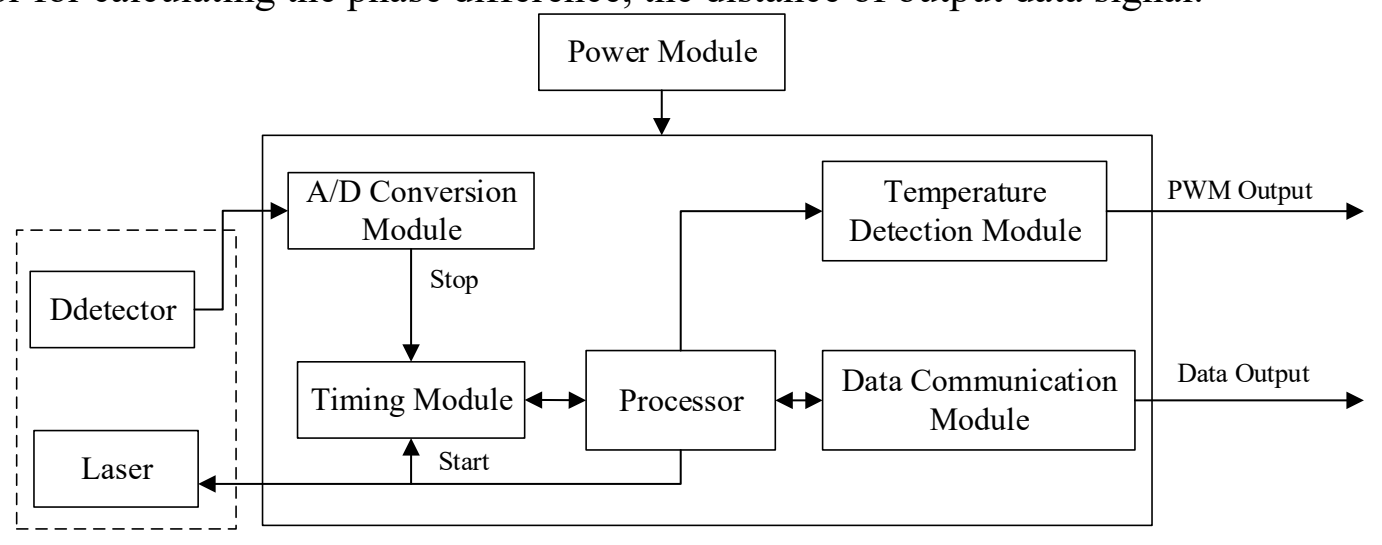

Figure 3 Signal processing flow

The information processing circuit board and the laser radar prototype are shown below.4-1 is A/D conversion module,4-2 is timing module,4-3 is processor,4-4 is data communication module,4-5 is data output module,4-6i is power module.

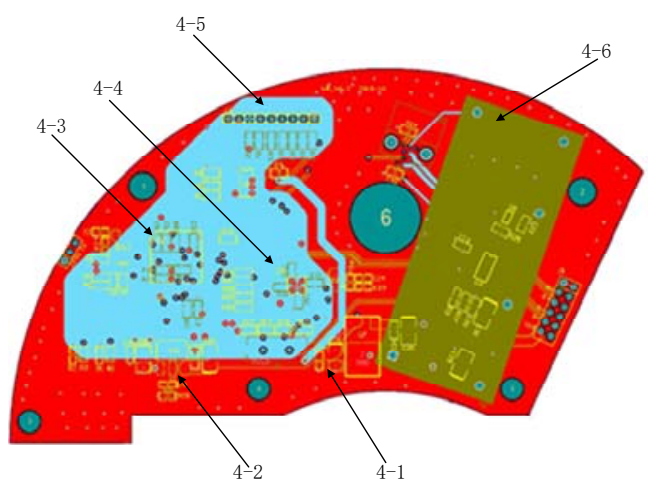

(a) Information processing circuit

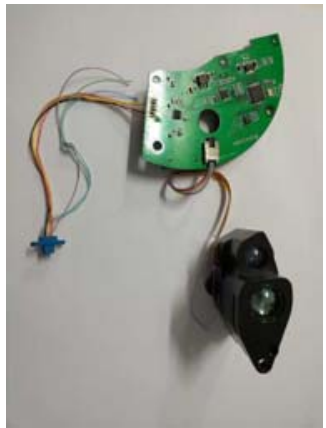

(b) laser radar prototype

Figure 4 Laser radar

\subsection{High Voltage Bias Circuit}

The direct voltage boost technique used in the laser radar can realize the $5 \sim 500 \mathrm{~V}$ DC output. It consists of three parts: boost circuit, back pressure circuit and temperature compensation, which provide the bias voltage for the laser component and detector according to the change of temperature. The direct step-up DC exchange with inductance can realize miniaturization and lightweight of the whole system, and improve the conversion efficiency.

The schematic diagram of high voltage offset power supply is shown in Figure 5. The boost circuit in the power module is a special boost module which can realize the function of DC/DC Converter. Through DC/DC Converter, the input voltage can be converted to a high voltage output of over 100 volts. The temperature compensation is the real-time temperature measured by DS18B20, and the 
control high voltage changes with the change of working temperature. If the temperature compensation is not allowed, the working voltage is insufficient or too large, which will greatly reduce the detection performance of laser radar.

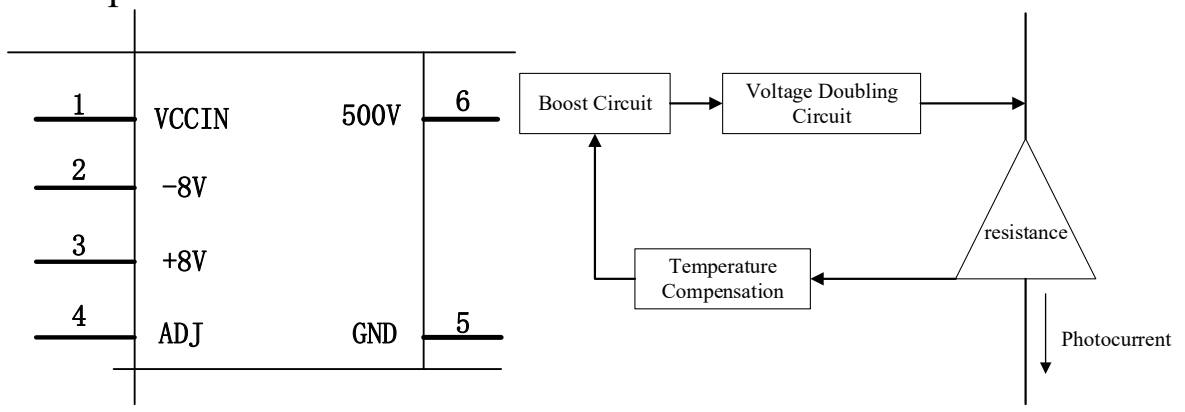

Figure 5 Schematic diagram of high voltage bias

\subsection{Principle of Phase Laser Radar Ranging}

Phase distance measurement, also called beam modulation telemetry. The phase laser radar range is used to transmit the modulated continuous wave to the target, and the distance measured by the phase difference between the reflected wave and the transmitted wave is measured to measure the distance to be measured. Phase laser radar ranging is the phase difference between the transmitted modulated light and the received light reflected by the target ${ }^{[7,8]}$.

The modulated wave of light wave can be represented by cosine expression, It is assumed here that the expression of the modulated wave is

$$
x(t)=\sin (2 \pi f t)
$$

$f_{c}$ is sampling frequency, $\mathrm{N}$ is sampling rate points, $f=q \times \Delta f$, and $q$ is integer, $\Delta f$ is frequency resolution, The discrete sequences of Formula 1 are sampled as follows

$$
x(n)=\sin \left(2 \pi f n T_{c}\right)=\sin \left(\frac{2 \pi}{N} \times q \times n\right)
$$

By Fast Fourier Transform can obtain

$$
X(k)=\sum_{n=0}^{N-1} x(n) W_{N}^{n k} \quad k=0,1,2 \ldots N-1
$$

For

$$
x(n) W_{N}^{n k}=\sin \left(\frac{2 \pi}{N} \cdot q \cdot n\right) e^{-j \frac{2 \pi}{N} n k}
$$

Can get

$$
X(k)=\frac{1}{2} j \sum_{n=0}^{N-1}\left\{\exp \left[-j \frac{2 \pi}{N}(q+k) n\right]-\left[-j \frac{2 \pi}{N}(q-k) n\right]\right\}
$$

On account of $k \neq q, X(k)=0 ; k=q, X(k)=-\frac{1}{2} N j \cdot \exp (j \varphi)$.

So, we can know $X(k)$ will be 0 just only when $k \neq q$. Write the expression of $x(n)$ in $q$, the real part and imaginary part of $X(k)$ at $k=q$ can be calculated. The phase information can be obtained from the formula

$$
\varphi=\cos ^{-1}\left(-\frac{\mathrm{Re}}{\operatorname{Re}^{2}+\mathrm{Im}^{2}}\right)
$$

Then

$$
d=\frac{c \varphi}{4 \pi f}
$$

In this way, we get the range value of laser ranging based on FFT. 


\section{Voltage Matching Algorithm Based on Machine Learning}

Machine learning is the core of artificial intelligence, is the basic way to make the computer intelligent, and its application throughout the field of artificial intelligence ${ }^{[9]}$. In order to give full play to the performance of laser radar, to reduce the influence of temperature variation on detection performance, the introduction of power matching algorithm based on machine learning in intelligent power matching, in the judgment does not appear to have the highest voltage garbled case, to achieve maximum detection power. Naive Bayes algorithm is a classical algorithm used in machine learning $^{[10]}$.

Take 100 numbers as a combination, and take $\mathrm{n}$ garbled data and normal data. For each combination of $x$ by conjunctive attribute description. And the objective function $f(x)$ is taken from a finite set $\mathrm{V}$. The learner is provided with a series of training examples and new examples about the objective function $\left\langle a_{1}, a_{2}, \ldots . a_{n}\right\rangle$. Then, the target value of the new instance is predicted, and the most probable target value is obtained $v_{\text {MAP }}$

$$
v_{\text {MAP }}=\arg \max _{v_{j} \in V} p\left(v_{j} \mid a_{1}, a_{2}, \ldots a_{n}\right)
$$

Using the Bayes formula, the expression can be rewritten as

$$
\begin{aligned}
v_{M A P} & =\arg \max _{v_{j} \in V} \frac{p\left(v_{j} \mid a_{1}, a_{2}, \ldots a_{n}\right) P\left(v_{j}\right)}{P\left(a_{1}, a_{2}, \ldots a_{n}\right)} \\
& =\arg \max _{v_{j} \in V} p\left(v_{j} \mid a_{1}, a_{2}, \ldots a_{n}\right) P\left(v_{j}\right)
\end{aligned}
$$

The naive Bias classifier is based on a simple assumption: when the given target value, the attribute values are mutually independent. Therefore, the probability of the joint $a_{1}, a_{2}, \ldots a_{n}$ is equal to the product of the probabilities of each individual attribute

$$
P\left(a_{1}, a_{2}, \ldots a_{n} \mid v_{j}\right)=\coprod_{i} P\left(a_{i} \mid v_{j}\right)
$$

Bring it to (10)

$$
v_{N B}=\arg \max _{v_{j} \in V} P\left(v_{j}\right) \prod_{i} P\left(a_{i} \mid v_{j}\right)
$$

$v_{N B}$ represents the output target value of the naive Bias classifier. For the given lidar data to be classified, the probability of the occurrence of each category in this condition is calculated, which is the largest, which category is considered to belong to.

In judging the data is normal when the data, in each traversal cycle gradually increased until the voltage, determine the new data classification is garbled data, take the traversal of the last voltage working voltage, to achieve the maximum working capacity of laser module. 


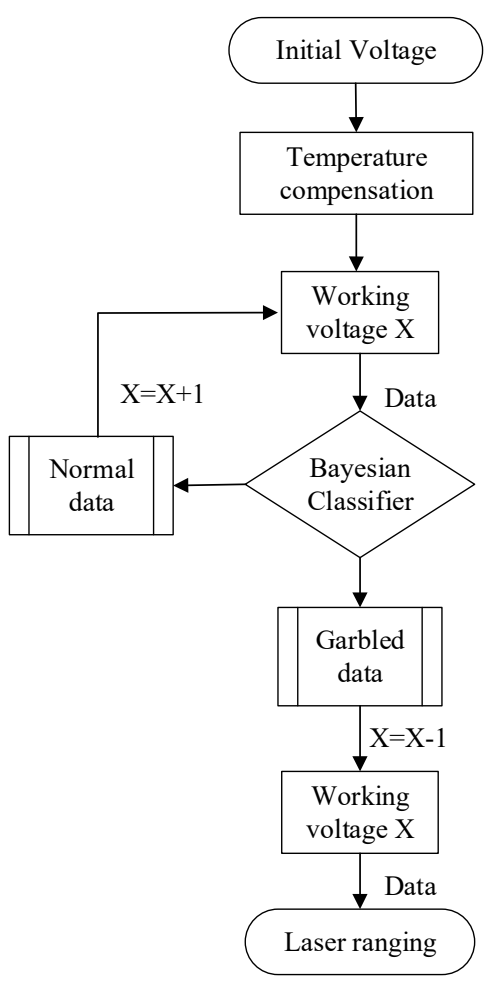

Figure 6 Ergodic voltage flow based on machine learning

\section{Experiment}

In order to verify the performance of the designed laser radar, functional tests were carried out on the prototype developed.

\subsection{Functional Test}

In the 16 floor of the building, the prototype is installed on the turntable, and the scanning path is determined by the coaxial laser pen, and then the target of $1.5 \times 2 \times 3 \mathrm{~m}^{3}$ is built on the scanning circle. As shown in Figure 7, 1 represents the height data inside the flower beds of flowers, 2 is a telegraph pole, 3 is the target to be measured.

References are cited in the text just by square brackets [1]. Two or more references at a time may be put in one set of brackets $[3,4]$. The references are to be numbered in the order in which they are cited in the text and are to be listed at the end of the contribution under heading references, see our example below.

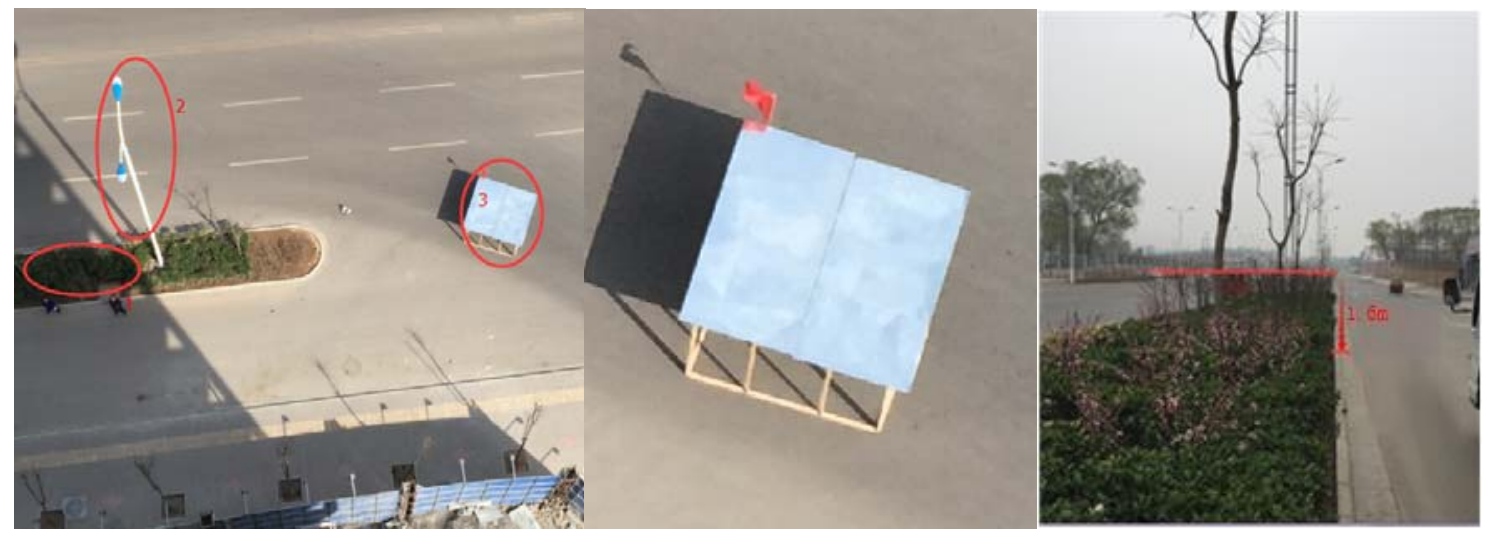

Figure 7 Test environment and target

The period data at the speed of $6.5 \mathrm{~s} / \mathrm{r}$ are as follows:

1 represents the height data inside the flower beds of flowers, 2 represents pole height data, 3 is the height data of the target to be measured. Zoom in on the target, as shown in the left picture below, 
the target can be clearly distinguished by 1.5 , and the jitter is about 0.1 meters. It is proved that the designed laser radar can accurately measure the distance between the target and the object in the dynamic environment.
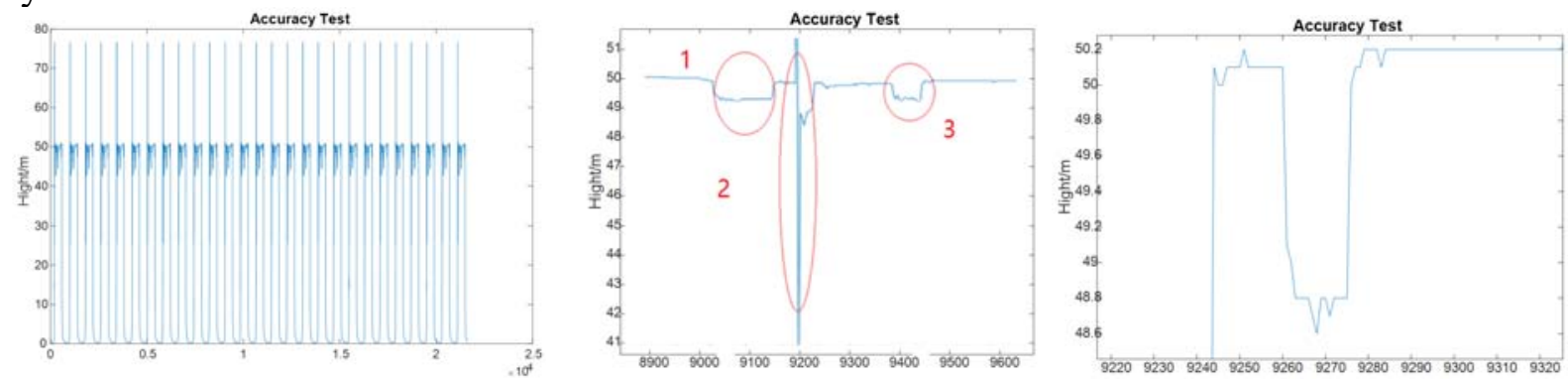

Figure 8 Test results

\subsection{Voltage Matching Algorithm and Accuracy Test}

The test environment is shown in Figure 9, measuring the distance of 300 meters with a ruler in the middle of the road, the laser radar, oscilloscope, DC power supply, notebook and so placed in the test end, the other side of the car is a target for the remote measurement.

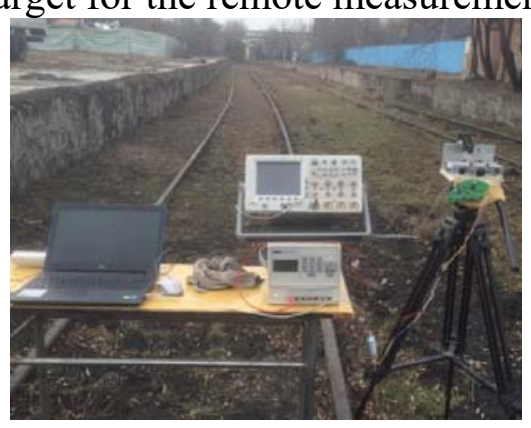

Figure 9 Test environment

The test results are shown in Figure 10, (a) for the first test method, the measurement results can clearly see no data, indicating that it is difficult to complete the $300 \mathrm{~m}$ ranging capability without adjusting voltage. (b) represent the increased voltage, and you can clearly see, a lot of garbled in the realization of the ranging capability, cannot be judged at the valid data, and manually adjust the dithering problem, difficult to accurately adjust the voltage, $300 \mathrm{~m}$ ranging. (c) is a voltage matching algorithm based on machine learning. The test results show that the proposed algorithm can measure the $300 \mathrm{~m}$ distance limited, and the ranging accuracy is $<0.1 \%$, which proves the correctness and scientificity of the proposed algorithm. (d) is the echo signal that measured under (c) condition. 


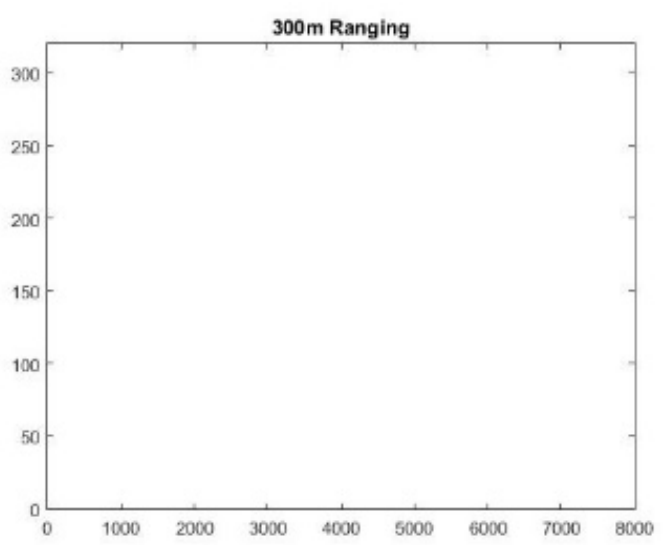

(a) Test diagram of initial voltage

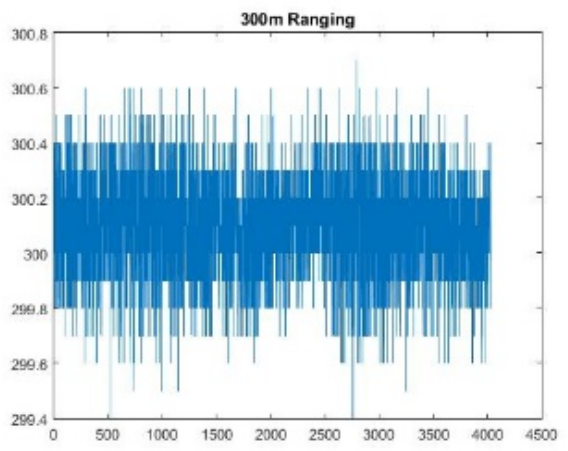

(c) Voltage matching test

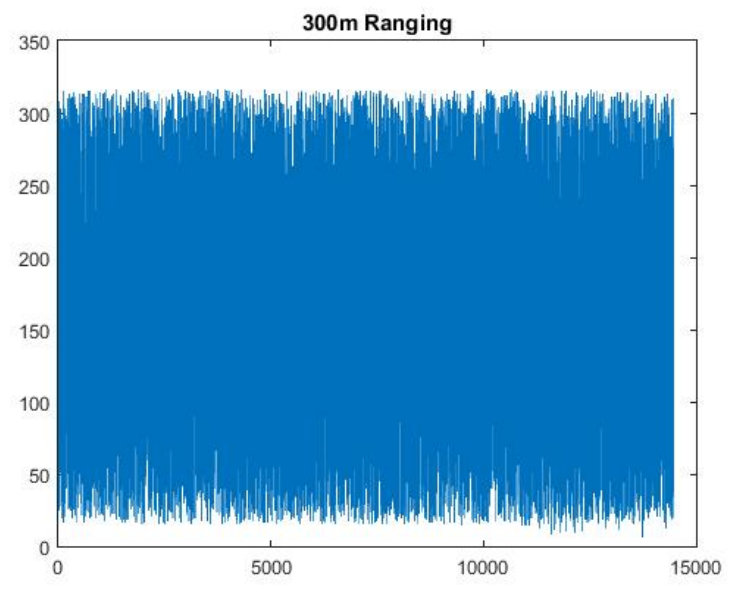

(b) Manual voltage regulation test

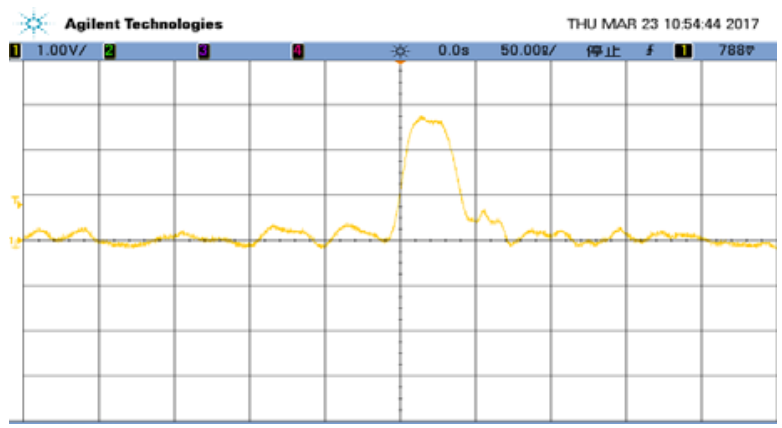

(d) Oscilloscope echo map

Figure 10 Test results

\section{Conclusions}

Through the accuracy test and voltage matching test, it is proved that the proposed laser radar can accurately measure long range target, has the ability of $300 \mathrm{~m}$ ranging, and fluctuates at $+0.3 \mathrm{~m}$ at 300 ranging, and the ranging accuracy is $<0.1 \%$. It is proved that the proposed voltage matching algorithm based on machine learning is correct and scientific, which can effectively compensate the adverse effects caused by temperature changes, and improve the ranging accuracy and ranging distance.

\section{Acknowledgments}

This work is supported by National Natural Science Foundation of China (Normal Project No. 61672093; Key Project No.61432004) and National Key Research \& Development Plan of China (No. 2016YFB1001404)..

\section{References}

[1] Subramanian V, Burks T F, Arroyo A A. Development of machine vision and laser radar based autonomous vehicle guidance systems for citrus grove navigation[J]. Computers and electronics in agriculture, 2006, 53(2): 130-143.

[2] Suzuki A J, Mizui K. Laser radar and visible light in a bidirectional V2V communication and ranging system[C]//Vehicular Electronics and Safety (ICVES), 2015 IEEE International Conference on. IEEE, 2015: 19-24. 
[3] Cheng Y Y, Tseng T, Chang K I, et al. Pulse radar ranging apparatus and ranging algorithm thereof: U.S. Patent 9,239,377[P]. 2016-1-19.

[4] Heintze M C, Munch J, Veitch P J. Development of a $1.5 \mu \mathrm{m}$ Er: Yb: glass laser for use in a Coherent Laser Radar[C]//Lasers and Electro-Optics, 2007. CLEO 2007. Conference on. IEEE, 2007: $1-2$.

[5] Nezhad M P, Simic A, Bondarenko O, et al. Room-temperature subwavelength metallo-dielectric lasers[J]. Nature Photonics, 2010, 4(6): 395-399.

[6] $\mathrm{Wu} \mathrm{X}$, Zhang A, Tong Z, et al. High precision laser ranging by combining phase method and multi-cycle pulse method[C]//Optical Communications and Networks (ICOCN 2011), 10th International Conference on. IET, 2011: 1-2.

[7] Jiang H, Lai J, Wang C, et al. Research on ranging property of laser radar and its range accuracy[J]. Zhongguo Jiguang(Chinese Journal of Lasers), 2011, 38(5): 0509001-7.

[8] Allodi M, Broggi A, Giaquinto D, et al. Machine learning in tracking associations with stereo vision and lidar observations for an autonomous vehicle[C]//Intelligent Vehicles Symposium (IV), 2016 IEEE. IEEE, 2016: 648-653.

[9] Kang Z, Yang J, Zhong R. A Bayesian-Network-Based Classification Method Integrating Airborne LiDAR Data With Optical Images[J]. IEEE Journal of Selected Topics in Applied Earth Observations and Remote Sensing, 2017, 10(4): 1651-1661.

[10] Dewan A, Oliveira G L, Burgard W. Deep Semantic Classification for 3D LiDAR Data[J]. arXiv preprint arXiv:1706.08355, 2017. 